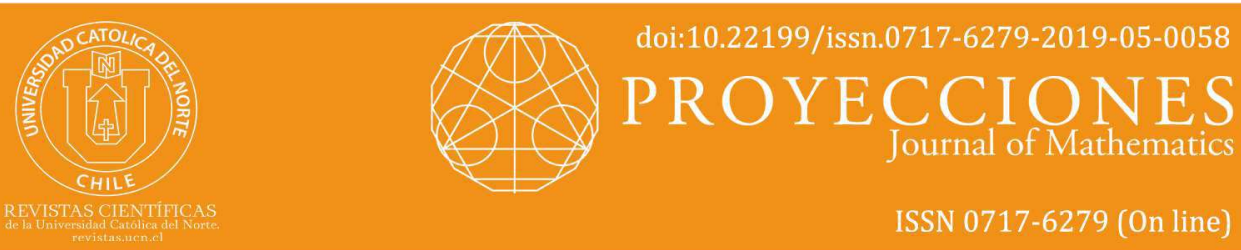

\title{
Left and right generalized Drazin invertible operators and local spectral theory
}

Mohammed Benharrat* (iD) orcid.org/0000-0002-2611-3391

Kouider Miloud Hocine**

Bekkai Messirdi $^{* * *}$ (iD) orcid.org/0000-0002-0155-000X

*Ecole Nationale Polytechnique d'Oran-Maurice Audin, Départ.de Mathématiques et Informatique, LMFAO, Oran, Algérie. $\nabla$ benharrat@math.univ-lyon1.fr

**Université des Sciences et de la Technologie d'Oran Mohamed-Boudiaf, Départ, de Mathématiques, LMFO, Oran, Algérie. $\nabla$ miloud.houcinek@gmail.com

${ }^{* * *}$ Ecole Supérieure en Génie électrique et energétique d'Oran, LMFAO, Oran, Algé-

rie. messirdi.bekkai@univ-oran.dz

\section{Received: August 2018 | Accepted: January 2019}

\section{Abstract:}

In this paper, we give some characterizations of the left and right generalized Drazin invertible bounded operators in Banach spaces by means of the single-valued extension property (SVEP). In particular, we show that a bounded operator is left (resp. right) generalized Drazin invertible if and only if admits a generalized Kato decomposition and has the SVEP at $O$ (resp. it admits a generalized Kato decomposition and its adjoint has the SVEP at 0 . In addition, we prove that both of the

Keywords: Left and right generalized Drazin invertible operators; Generalized Drazin invertible operators; SVEP; Local spectral theory.ions.

MSC (2010): 47A10.

\section{Cite this article as (IEEE citation style):}

M. Benharrat, K. Hocineand and B. Messirdi, "Left and right generalized Drazin invertible operators and local spectral theory", Proyecciones (Antofagasta, On line), vol. 38, no. 5, pp. 897-919, Dec. 2019, doi: 10.22199/issn.0717-6279-201905-0058. [Accessed dd-mm-yyyy].

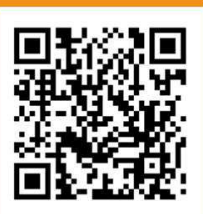

Article copyright: (C) 2019 Mohammed Benharrat. Kouider Miloud Hocine and Bekkai Messirdi. This is an open access article distributed under the terms of the Creative Commons Licence, which permits unrestricted use and distribution provided the original author and source are credited.

(cc) BY 


\section{Introduction}

The generalized Drazin inverse for operators arises naturally in the context of isolated spectral points and becomes a theoretical and practical tool in algebra and analysis (Markov chains, singular differential and difference equations, iterative methods...), see $[3,4,13,15,16]$ and the references therein. The Drazin inverse was originally defined in 1958 for semigroups ([6]). When $\mathcal{B}(X)$ is the Banach algebra of all bounded linear operators acting on an infinite-dimensional complex Banach space $X$, then $S \in \mathcal{B}(X)$ is the Drazin inverse of $T \in \mathcal{B}(X)$ if

$S T=T S \quad S T S=S$ and $T S T=T+U$ where $U$ is a nilpotent operator. (1.1)

The concept of Drazin invertible operators has been generalized by Koliha ([14]) by replacing the nilpotent operator $U$ in defDrazin by a quasinilpotent operator. In this case, $S$ is called a generalized Drazin inverse of $T$. Note that this extension was anticipated by Harte in [10]. Recently, in [20], the authors introduced the left and the right generalized Drazin invertible operators. These two classes of operators are a continuation and refinement of the research treatment of the Drazin inverse in Banach space operators theory. It proved that an operator $T \in \mathcal{B}(X)$ is left (resp. right) generalized Drazin invertible if and only if $T=T_{1} \oplus T_{2}$ where $T_{1}$ is bounded below (resp. surjective) and $T_{2}$ is quasinilpotent operator. Furthermore, these operators are characterized via the isolated points of the approximate point spectrum (resp. surjective spectrum) [20, Theorem 3.8; Theorem 3.10].

The purpose of this paper is to study the relationship between local spectral properties of a bounded operator and its left and right generalized inverses. In Section 2, we give some preliminary results which our investigation will be need. In Section 3, we present many new and interesting characterizations of the left (resp. the right) generalized Drazin invertible operators in terms of the generalized Kato decomposition and the single-valued extension property. We also show that an operator admits a generalized Kato decomposition and has the SVEP at 0 is precisely left generalized Drazin invertible and conversely. Similarly, an operator $T$ is right generalized Drazin invertible if and only if $T$ admits a generalized Kato decomposition and its adjoint $T^{*}$ has the SVEP at 0. In particular, we prove that the left generalized Drazin spectrum and right generalized Drazin spectrum of a bounded operator are invariant under commuting finite rank perturbations. In section 4 , we study the relationships between 
the local spectral properties of an operator and the local spectral properties of its generalized Drazin inverse, if this exists. In particular, a reciprocal relationship analogous to spectrum of invertible operator and its inverse, is established between the nonzero points of the local spectrum of a generalized Drazin invertible operator having SVEP and the nonzero points of the local spectrum of its generalized Drazin inverse. We also show that many local spectral properties, as SVEP, Dunford property $(C)$, property $(\beta)$, property $(Q)$ and decomposability, are transferred from a generalized Drazin invertible operator to its generalized Drazin inverse. This section extends the results of [2] from the case of Drazin invertible operators to case of the generalized Drazin invertible operators. Finally, by a counterexample we show that these local spectral properties are not transferred in the case of the left (resp. the right) generalized Drazin invertible operators.

\section{Preliminaries}

Let $\mathcal{B}(X)$ be the Banach algebra of all bounded linear operators acting on an infinite-dimensional complex Banach space $X$.

For $T \in \mathcal{B}(X)$ write $N(T), R(T), \sigma(T)$ and $\rho(T)$ respectively, the null space, the range, the spectrum and the resolvent set of $T$. The nullity and the deficiency of $T$ are defined respectively by $\alpha(T)=\operatorname{dim} N(T)$ and $\beta(T)=\operatorname{dim} X / R(T)$. Here $I$ denote the identity operator in $X$. By iso $\sigma(T)$ and $\operatorname{acc} \sigma(T)$ we define the set of all isolated and accumulation spectral points of $T$.

If $M$ is a subspace of $X$ then $T_{M}$ denotes the restriction of $T$ in $M$. Assume that $M$ and $N$ are two closed subspaces of $X$ such that $X=M \oplus N$ (that is, $X=M+N$ and $M \cap N=0$ ). We say that $T$ is completely reduced by the pair $(M, N)$, denoted as $(M, N) \in \operatorname{Red}(T)$, if $T(M) \subset M, T(N) \subset N$ and $T=T_{M} \oplus T_{N}$. In such case we have $N(T)=N\left(T_{M}\right) \oplus N\left(T_{N}\right)$, $R(T)=R\left(T_{M}\right) \oplus R\left(T_{N}\right)$, and $T^{n}=T_{M}^{n} \oplus T_{N}^{n}$ for all $n \in \mathbf{N}$. An operator is said to be bounded below if it is injective with closed range.

Recall that (see, e.g. [11]) the ascent $a(T)$ of an operator $T \in \mathcal{B}(X)$ is defined as the smallest nonnegative integer $p$ such that $N\left(T^{p}\right)=N\left(T^{p+1}\right)$. If no such integer exists, we set $a(T)=\infty$. Analogously, the smallest nonnegative integer $q$ such that $R\left(T^{q}\right)=R\left(T^{q+1}\right)$ is called the descent of $T$ and denoted by $d(T)$. We set $d(T)=\infty$ if for each $q, R\left(T^{q+1}\right)$ is a proper subspace of $R\left(T^{q}\right)$. It is well known that if the ascent and the descent of an operator are finite, then they are equal.

Associated with an operator $T \in \mathcal{B}(X)$ there are two (not necessarily 
closed) linear subspaces of $X$ invariant by $T$, played an important role in the development of the generalized Drazin inverse for $T$, the quasinilpotent part $H_{0}(T)$ of $T$ :

$$
H_{0}(T)=\left\{x \in x: \lim _{n \rightarrow \infty}\left\|T^{n} x\right\|^{\frac{1}{n}}=0\right\},
$$

and the analytical core $K(T)$ of $T$ :

$K(T)=\left\{x \in X:\right.$ there exist a sequence $\left(x_{n}\right)$ in $X$ and a constant $\delta>0$

such that $T x_{1}=x, T x_{n+1}=x_{n}$ and $\left\|x_{n}\right\| \leq \delta^{n}\|x\|$ for all $\left.n \in \mathbf{N}\right\}$.

It is well-known that necessary and sufficient condition for $T \in \mathcal{B}(X)$ to be generalized Drazin invertible is that $0 \notin \operatorname{acc} \sigma(T)$. Equivalently, $K(T)$ and $H_{0}(T)$ are both closed, $X=H_{0}(T) \oplus K(T)$, the restriction of $T$ to $H_{0}(T)$ is a quasinilpotent operator, and the restriction of $T$ to $K(T)$ is invertible,

Recently, by the use of this two subspaces, in [20], the authors defined and studied a new class of operators called left and right generalized Drazin invertible operators as a generalization of left and right Drazin invertible operators.

Definition 2.1. An operator $T \in \mathcal{B}(X)$ is said to be right generalized Drazin invertible if $K(T)$ is closed and complemented with a subspace $N$ in $X$ such that $T(N) \subset N \subseteq H_{0}(T)$.

Definition 2.2. An operator $T \in \mathcal{B}(X)$ is said to be left generalized Drazin invertible if $H_{0}(T)$ is closed and complemented with a subspace $M$ in $X$ such that $T(M) \subset M$ and $T(M)$ is closed.

We have,

Proposition 2.3. Let $T \in \mathcal{B}(X)$. Then $T$ is left generalized Drazin invertible if and only if $T=T_{1} \oplus T_{2}$ such that $T_{1}$ is bounded below and $T_{2}$ is quasinilpotent.

Proof. Let $T \in B(X)$ be left generalized Drazin invertible, then $H_{0}(T)$ is closed and complemented with a subspace $M$ in $X$ such that $T(M)$ is closed. Then $T=T_{M} \oplus T_{H_{0}(T)}$, it is clear that $T_{H_{0}(T)}$ is quasinilpotent. Furthermore, we have $N\left(T_{M}\right)=M \cap N(T) \subseteq M \cap H_{0}(T)=\{0\}$, this means that $T_{M}$ is injective and since $T(M)$ is closed it follows that $T_{M}$ is bounded below. We obtain the result by taking $T_{1}=T_{M}$ and $T_{2}=T_{H_{0}(T)}$. 
Now, we prove the inverse. Suppose that $T=T_{1} \oplus T_{2}$ such that $T_{1}$ is left invertible and $T_{2}$ is quasinilpotent, then there are two subspaces $X_{1}$ and $X_{2}$ of $X$ such that $\left(X_{1}, X_{2}\right) \in \operatorname{Red}(T)$ with $X_{2}$ is closed. Let $T_{1}=T_{X_{1}}$ and $T_{2}=T_{X_{2}}$. Since $T_{2}$ is quasinilpotent clearly $H_{0}\left(T_{X_{2}}\right)=X_{2}$. On the other hand, $T_{X_{1}}$ is bounded below then $H_{0}\left(T_{X_{1}}\right)=\{0\}$, hence $H_{0}(T)=$ $H_{0}\left(T_{X_{1}}\right) \oplus H_{0}\left(T_{X_{2}}\right)=H_{0}\left(T_{X_{2}}\right)=X_{2}$, this shows that $X=X_{1} \oplus H_{0}(T)$ with $H_{0}(T)$ and $T\left(X_{1}\right)$ are closed in $X$.

Proposition 2.4. Let $T \in \mathcal{B}(X)$. Then $T$ is right generalized Drazin invertible if and only if $T=T_{1} \oplus T_{2}$ such that $T_{1}$ is surjective and $T_{2}$ is quasinilpotent.

Proof. If $T \in B(X)$ is right generalized Drazin invertible, then $K(T)$ is closed and complemented in $X$ with a subspace $N \subseteq H_{0}(T)$. Let $T_{1}=$ $T_{K(T)}$ and $T_{2}=T_{N}$, then $T=T_{1} \oplus T_{2}$. We have $R\left(T_{1}\right)=R(T) \cap K(T)=$ $K(T)$, it follows that $T_{1}$ is surjective. Also, we have $H_{0}\left(T_{2}\right)=H_{0}(T) \cap N=$ $N$, thus $T_{2}$ is quasinilpotent.

Conversely, if $T=T_{1} \oplus T_{2}$ with $T_{1}$ is surjective and $T_{2}$ is quasinilpotent, then there are two subspaces $X_{1}$ and $X_{2}$ of $X$ such that $\left(X_{1}, X_{2}\right) \in \operatorname{Red}(T)$. By [1, Theorem 1.41], we deduce that $K\left(T_{X_{1}}\right)=K(T)$ is closed. Since $T_{X_{1}}$ is surjective, $T\left(X_{1}\right)=X_{1}$, hence $X_{1} \subset K(T)$. On the other hand, we have $K(T)=K\left(T_{X_{1}}\right) \subset T\left(X_{1}\right)=X_{1}$ which implies that $K(T)=X_{1}$, it follows that $X=K(T) \oplus X_{2}$. The inclusion $X_{2} \subseteq H_{0}(T)$ follows by the the fact that $T_{X_{2}}$ is quasinilpotent.

As a corollary, in the Hilbert space, we have the following result.

Corollary 2.5. $T \in \mathcal{B}(X)$ and $X$ is an Hilbert space, we have

1. $T$ is left generalized Drazin invertible if and only if $T=T_{1} \oplus T_{2}$ such that $T_{1}$ is left invertible and $T_{2}$ is quasinilpotent.

2. $T$ is right generalized Drazin invertible if and only if $T=T_{1} \oplus T_{2}$ such that $T_{1}$ is right invertible and $T_{2}$ is quasinilpotent.

Examples of left generalized Drazin invertible operators are the operators of the following classes:

- Left invertible operators on Hibert space.

- Left Drazin invertible operators such that $H_{0}(T)=N\left(T^{p}\right)(a(T)=$ $p<\infty)$ is complemented with a $T$-invariant subspace $M$ for which $T(M)$ is closed. 
- Drazin invertible operators.

- A bounded paranormal operator $T$ on Hilbert space $X$ such that $R(T)+H_{0}(T)$ and $H_{0}(T)$ are closed (see [20, Proposition 3.15]).

Examples of right generalized Drazin invertible operators are the operators of the following classes:

- Right invertible operators on Hilbert space.

- Right Drazin invertible operators, such that $K(T)=R\left(T^{q}\right)$ (with $d(T)=q<\infty)$ is complemented by a closed $T$-invariant subspace $N$ with $N \subseteq H_{0}(T)$.

- Drazin invertible operators.

According to the Definitions 2.1 and 2.2, we also have

Invertible operator $\Longrightarrow$ Generalized Drazin invertible operator $\Longrightarrow$ Right (resp. Left) generalized Drazin invertible operator.

In the sequel the terms left (resp. right) generalized Drazin invertible operator is used for the nontrivial case of the bounded below (resp. surjective) operators.

The left Drazin spectrum, the right Drazin spectrum, the Drazin spectrum, the generalized Drazin spectrum, the left generalized Drazin spectrum and the right generalized Drazin spectrum of $T$ are, respectively, defined by

$\sigma_{l D}(T):=\{\lambda \in \mathbf{C}: \lambda I-T$ is not left Drazin invertible $\}$,

$\sigma_{r D}(T):=\{\lambda \in \mathbf{C}: \lambda I-T$ is not right generalized Drazin invertible $\}$,

$\sigma_{D}(T)=\{\lambda \in \mathbf{C}: \lambda I-T$ is not Drazin invertible $\}$,

$\sigma_{g D}(T)=\{\lambda \in \mathbf{C}: \lambda I-T$ is not generalized Drazin invertible $\}$

$\sigma_{l g D}(T):=\{\lambda \in \mathbf{C}: \lambda I-T$ is not left generalized Drazin invertible $\}$,

and

$\sigma_{r g D}(T):=\{\lambda \in \mathbf{C}: \lambda I-T$ is not right generalized Drazin invertible $\}$. 
It is well known that these spectra are compact sets in the complex plane, and we have,

$$
\begin{gathered}
\sigma_{g D}(T)=\sigma_{l g D}(T) \cup \sigma_{r g D}(T) \subset \sigma_{D}(T)=\sigma_{l D}(T) \cup \sigma_{r D}(T), \\
\sigma_{l g D}(T) \subset \sigma_{a p}(T)
\end{gathered}
$$

and

$$
\sigma_{r g D}(T) \subset \sigma_{s u}(T)
$$

where

$$
\sigma_{a p}(T):=\{\lambda \in \mathbf{C}: \lambda I-T \text { is not bounded below }\}
$$

and

$$
\sigma_{s u}(T):=\{\lambda \in \mathbf{C}: \lambda I-T \text { is not surjective }\},
$$

are respectively the approximate point spectrum and the surjective spectrum of $T$.

An operator $T \in \mathcal{B}(X), T$ is said to be semi-regular if $R(T)$ is closed and $N\left(T^{n}\right) \subseteq R(T)$, for all $n \in \mathbf{N}$. An important class of operators which involves the concept of semi-regularity is the class of operators admits a generalized Kato decomposition.

Definition 2.6. (see [1]) $T \in \mathcal{B}(X)$ is said to admit a generalized Kato decomposition, abbreviated as GKD, if there exists a pair of closed subspaces $(M, N)$ such that $(M, N) \in \operatorname{Red}(T)$ with $T_{M}$ is semi-regular and $T_{N}$ is quasinilpotent.

The pair $(M, N)$ is called the generalized Kato decomposition of $T$ and denoted by $\operatorname{GKD}(M, N)$.

If we assume in the definition above that $T_{N}$ is nilpotent, then there exists $d \in \mathbf{N}$ for which $\left(T_{N}\right)^{d}=0$. In this case $T$ is said to be of Kato type operator of degree $d$. Examples of operators admits a generalized Kato decomposition, are Kato type operators, semi-regular operators, semiFredholm operators, quasi-Fredholm operators and generalized Drazin invertible operators, some other examples may be found in [17].

Let $M$ be a subspace of $X$ and let $X^{*}$ be the dual space of $X$. As it is usual, $M^{\perp}=\left\{x^{*} \in X^{*}: x^{*}(M)=0\right\}$. Moreover, if $M$ and $N$ are closed linear subspaces of $X$ then $(M+N)^{\perp}=M^{\perp} \cap N^{\perp}$. The dual relation 
$M^{\perp}+N^{\perp}=(M \cap N)^{\perp}$ is not always true, since $(M \cap N)^{\perp}$ is always closed but $M^{\perp}+N^{\perp}$ need not be closed. However, a classical theorem establishes that $M^{\perp} \cap N^{\perp}$ is closed in $X^{*}$ if and only if $M+N$ is closed in $X$, (see $[12$, Theorem 4.8, Chapter IV]).

Theorem 2.7 ([1]). Let $T \in \mathcal{B}(X)$. If $(M, N)$ is a $G K D$ of $T$, then $\left(N^{\perp}, M^{\perp}\right)$ is a $G K D$ of its adjoint $T^{*}$. Furthermore, if $T$ is of a Kato type operator then $T^{*}$ is also of a Kato type.

For every operator $T \in \mathcal{B}(X)$, let us define the semi-regular spectrum, the Kato spectrum and the generalized Kato spectrum as follows:

$$
\begin{aligned}
& \sigma_{s e}(T):=\{\lambda \in \mathbf{C}: \lambda I-T \text { is not semi-regular }\} \\
& \sigma_{k}(T):=\{\lambda \in \mathbf{C}: \lambda I-T \text { is not of Kato type }\} \\
& \sigma_{g k}(T):=\{\lambda \in \mathbf{C}: \lambda I-T \text { does not admit a generalized Kato decomposition }\}
\end{aligned}
$$

Recall that all the three sets defined above are always compact subsets of the complex plane, (see [1], [7]) and ordered by :

$$
\sigma_{g k}(T) \subseteq \sigma_{k}(T) \subseteq \sigma_{s e}(T) .
$$

Furthermore, the generalized Kato spectrum of an operator differs from the semi-regular spectrum on at most countably many isolated points, more precisely the sets $\sigma_{s e}(T) \backslash \sigma_{g k}(T), \sigma_{s e}(T) \backslash \sigma_{k}(T)$ and $\sigma_{k}(T) \backslash \sigma_{g k}(T)$ are at most countable (see [1] and [7]).

Note that $\sigma_{g k}(T)$ (resp. $\sigma_{k}(T)$ ) is not necessarily non-empty. For example, a quasinilpotent (resp. nilpotent) operator $T$ has empty generalized Kato spectrum (resp. Kato spectrum). Furthermore, the comparison between this spectra and the spectra defined by the Drazin inverses gives

$$
\sigma_{g k}(T) \subset \sigma_{l g D}(T) \subset \sigma_{a p}(T),
$$

and

$$
\sigma_{g k}(T) \subset \sigma_{r g D}(T) \subset \sigma_{s u}(T) .
$$

Definition 2.8. Let $T \in \mathcal{B}(X)$. The operator $T$ is said to have the singlevalued extension property at $\lambda_{0} \in \mathbf{C}$, abbreviated $T$ has the SVEP at $\lambda_{0}$, if for every neighborhood $\mathcal{U}$ of $\lambda_{0}$ the only analytic function $f: \mathcal{U} \rightarrow X$ which satisfies the equation

$$
(\lambda I-T) f(\lambda)=0
$$


is the constant function $f \equiv 0$.

The operator $T$ is said to have the SVEP if $T$ has the SVEP at every $\lambda \in \mathbf{C}$.

Trivially, an operator $T$ has the SVEP at every point of the resolvent set $\rho(T)$. Moreover, from the identity theorem for analytic functions it easily follows that $T$ has the SVEP at every point of the boundary $\partial \sigma(T)$ of the spectrum. Hence, we have the implications:

1. Every operator $T$ has the SVEP at an isolated point of the spectrum.

2. If $\lambda \notin \operatorname{acc} \sigma_{a p}(T)$, then $T$ has the SVEP at $\lambda$.

3. If $\lambda \notin \operatorname{acc} \sigma_{s u}(T)$, then $T^{*}$ has the SVEP at $\lambda$

In particular, it has been showed that if $\lambda I-T$ admits a generalized Kato decomposition, then implications (2) and (3) may be reversed. For more properties of the SVEP, we can see [19].

\section{Left and right generalized Drazin invertible operators and the SVEP}

Now we give a characterization of the left (resp. the right) generalized Drazin invertible operators in terms of generalized Kato decomposition and the single-valued extension property.

Theorem 3.1. An operator $T \in \mathcal{B}(X)$ is left generalized Drazin invertible if and only if $T$ admits a $G K D(M, N)$ and $T_{M}$ has the SVEP at 0.

Proof. By definition 2.1 a left generalized Drazin invertible operator $T$ admits a $\operatorname{GKD}(M, N)$ with $H_{0}(T)=N$ is closed, hence $T$ has the SVEP at 0 . So, $T_{M}$ has the SVEP at 0 . Conversely, if $T$ admits a $\operatorname{GKD}(M, N)$ with $T_{M}$ has the SVEP at 0 . Then by [1, Theorem 3.14] $T_{M}$ is injective and $H_{0}(T)=N$. Since $R\left(T_{M}\right)$ is closed, $T_{M}$ is bonded below. Hence $T$ is left generalized Drazin invertible.

Dually, by Definition 2.2 and [1, Theorem 3.15], we get the following result,

Theorem 3.2. An operator $T \in \mathcal{B}(X)$ is right generalized Drazin invertible if and only if $T$ admits a GKD $(M, N)$ and $T^{*}$ has the SVEP at 0.

Again by [1, Theorem 3.14], there are an equivalent properties to $T_{M}$ has the SVEP at 0 for operators admits a GKD, so we can say more about the left generalized Drazin invertible operators. 
Theorem 3.3. An operator $T \in \mathcal{B}(X)$ is left generalized Drazin invertible if and only if $T$ admits a $G K D(M, N)$ and satisfies one of the following equivalent assertions:

(i) $T$ has the SVEP at 0 ,

(ii) $T_{M}$ has the SVEP at 0 ,

(iii) $T_{M}$ is injective,

(iv) $H_{0}(T)=N$,

(v) $H_{0}(T)$ is closed,

(vi) $K(T) \cap H_{0}(T)=\{0\}$,

(vii) $K(T) \cap H_{0}(T)$ is closed.

Similarly, by [1, Theorem 3.15] we have:

Theorem 3.4. An operator $T \in \mathcal{B}(X)$ is right generalized Drazin invertible if and only if $T$ admits a $G K D(M, N)$ and satisfies one of the following equivalent assertions:

(i) $T^{*}$ has the SVEP at 0 ,

(ii) $T_{M}$ is surjective,

(iii) $K(T)=M$,

(iv) $X=K(T)+H_{0}(T)$,

(v) $K(T)+H_{0}(T)$ is norm dense in $X$.

The following result expresses a characterization of the isolated points of $\sigma_{a p}(T)$ in terms of generalized Kato decomposition and the SVEP.

Proposition 3.5. Let $T \in \mathcal{B}(X)$ and $0 \in \sigma_{a p}(T)$. Then 0 is an isolated point in $\sigma_{a p}(T)$ if and only if $T$ admits a $\operatorname{GKD}(M, N)$ and $T_{M}$ has the SVEP at 0. 
Proof. Suppose that 0 is an isolated point in $\sigma_{a p}(T)$, then $T$ has the SVEP at 0 and by [9, Proposition 9.], $H_{0}(T)$ and $K(T)$ are closed subspaces of $X$ with $K(T) \neq X, H_{0}(T) \neq\{0\}$ and $K(T) \cap H_{0}(T)=\{0\}$. If $K(T) \oplus$ $H_{0}(T)=X$, then 0 is also isolated point in $\sigma(T)$ and clearly $T$ admits a $\operatorname{GKD}\left(K(T), H_{0}(T)\right)$. Now, assume that $K(T) \oplus H_{0}(T) X$. Let $X_{0}=$ $K(T) \oplus H_{0}(T)$ and $T_{0}: X_{0} \longrightarrow X_{0}$ the operator induced by $T$ on $X_{0}$. We have $X_{0}$ is a Banach space and $\left(K\left(T_{0}\right), H_{0}\left(T_{0}\right)\right)=\left(K(T), H_{0}(T)\right)$ is a GKD of $T$ on $X_{0}$. So by Theorem $2.7\left(H_{0}(T)^{\perp}, K(T)^{\perp}\right)$ is also a GKD of $T^{*}$ over $X_{0}^{*}=H_{0}(T)^{\perp} \oplus K(T)^{\perp}$. Here, $H_{0}(T)^{\perp}$ and $K(T)^{\perp}$ are the annihilators of $H_{0}(T)$ and $K(T)$ in $X_{0}^{*}$, respectively. In the other hand, we know that the adjoint of the inclusion map $i: X_{0} \rightarrow X$ is a map from $X^{*}$ onto $X_{0}^{*}$ with kernel $X_{0}^{\perp}$. This implies that $T^{*}$ admits a GKD over $X^{*}$. Again by Theorem 2.7 $T$ admits a $\operatorname{GKD}(M, N)$ viewed as a restriction of the adjoint of $T^{*}$ on $X$. Further, $T_{M}$ has the SVEP at 0 because the SVEP is inherited by the restrictions on invariant subspaces. Conversely, if $T$ admits a $\operatorname{GKD}(M, N)$ and $T_{M}$ has the SVEP at 0 . Then by $[1$, Theorem 3.14] $T_{M}$ is injective and $H_{0}(T)=N$. Since $R\left(T_{M}\right)$ is closed, $T_{M}$ is bonded below. Hence $T$ is left generalized Drazin invertible. By Proposition 2.30 is an isolated point in $\sigma_{a p}(T)$.

Proposition 3.6. Let $T \in \mathcal{B}(X)$ and $0 \in \sigma_{s u}(T)$. Then 0 is an isolated point in $\sigma_{s u}(T)$ if and only if $T$ admits a $\operatorname{GKD}(M, N)$ and $T_{N^{\perp}}^{*}$ has the SVEP at 0.

Proof. Since $\sigma_{s u}(T)=\sigma_{a p}\left(T^{*}\right)$, we apply Proposition 3.5 to the operator $T^{*}$; we obtain that 0 is an isolated point in $\sigma_{a p}\left(T^{*}\right)$ if and only if $T^{*}$ admits a $\operatorname{GKD}\left(N^{\perp}, M^{\perp}\right)$ and $T_{N^{\perp}}^{*}$ has the SVEP at 0 , for some two closed subspaces of $X$. Now by Theorem 2.7, we get the desired result.

The basic existence results of generalized Drazin inverses and their relation to the SVEP, the quasinilpotent part and the analytical core are summarized in the following theorems.

Theorem 3.7. Assume that $T \in \mathcal{B}(X)$. The following assertions are equivalent:

(i) $T$ is left generalized Drazin invertible,

(ii) $T=T_{1} \oplus T_{2}$, with $T_{1}=T_{M}$ is bounded below operator and $T_{2}=$ $T_{H_{0}(T)}$ is quasinilpotent operator,

(iii) 0 is at most an isolated point in $\sigma_{a p}(T)$, 
(iv) $T$ admits a $\operatorname{GKD}(M, N)$ and $T_{M}$ has the $S V E P$ at 0 ,

(v) $T$ admits a $\operatorname{GKD}(M, N)$ and verified one of the equivalent conditions of the Theorem 3.3,

(vi) there exists a bounded projection $P$ on $X$ such that $T P=P T, T+P$ is bounded below, $T P$ is quasinilpotent and $R(P)=H_{0}(T)$.

Proof. The equivalence $(\mathrm{i}) \Longleftrightarrow(\mathrm{vi})$ has been proved in [8, Theorem 3.1]. By Theorems 3.1 and 3.3, we have (i) $\Longleftrightarrow(\mathrm{v}) \Longleftrightarrow$ (iv) and (iv) $\Longleftrightarrow$ (iii) follows from Proposition 3.5. By what we assert that (i) $\Longleftrightarrow$ (iv) $\Longleftrightarrow$ (iii). Finally, (i) $\Longleftrightarrow$ (ii) follows from Proposition 2.3.

We know that the properties to be right generalized Drazin invertible or to be left generalized Drazin invertible are dual to each other, (see [20, Proposition 3.9]), then we have,

Theorem 3.8. Let $T \in \mathcal{B}(X)$. The following assertions are equivalent:

(i) $T$ is right generalized Drazin invertible,

(ii) $T=T_{1} \oplus T_{2}$, with $T_{1}=T_{K(T)}$ is surjective operator and $T_{2}=T_{N}$ is quasinilpotent operator,

(iii) 0 is at most an isolated point in $\sigma_{s u}(T)$,

(iv) $T$ admits a $\operatorname{GKD}(M, N)$ and $T^{*}$ has the $S V E P$ at 0 ,

(v) T admits a $\operatorname{GKD}(M, N)$ and satisfied one of the equivalent conditions of the Theorem 3.4,

(vi) there exists a bounded projection $P$ on $X$ such that $T P=P T, T+P$ is surjective, $T P$ is quasinilpotent and $N(P)=K(T)$.

Proof. Similarly, The equivalence $(\mathrm{i}) \Longleftrightarrow(\mathrm{vi})$ has been proved in [8, Theorem 3.2]. By Theorems 3.2 and 3.4, we have (i) $\Longleftrightarrow(\mathrm{v}) \Longleftrightarrow(\mathrm{iv})$ and (iv) $\Longleftrightarrow$ (iii) follows from Proposition 3.6. Thus, (i) $\Longleftrightarrow$ (vi) $\Longleftrightarrow(\mathrm{v}) \Longleftrightarrow$ (iv) $\Longleftrightarrow$ (iii). Finally, (i) $\Longleftrightarrow$ (ii) follows from Proposition 2.4.

Remark 3.9. Theorems 3.7 and 3.8 are improvement of [20, Theorem 3.8, Theorem 3.10].

A direct consequence of Theorem 3.7, Theorem 3.8 and [1, Lemma 3.13]: 
Corollary 3.10. Let $T \in \mathcal{B}(X)$.

- If $T$ is left generalized Drazin invetible, then $H_{0}(T)^{\perp}=K\left(T^{*}\right)$.

- If $T$ is right generalized Drazin invetible, then $K\left(T^{*}\right)=H_{0}\left(T^{*}\right)^{\perp}$.

- If $T$ is left generalized Drazin invetible, then $T^{*}$ is a right generalized Drazin invetible with $N\left(P^{*}\right)=K\left(T^{*}\right)=H_{0}\left(T^{*}\right)^{\perp}=R(P)^{\perp}$, where $P$ is the bounded projection given in Theorem 3.7-(vi).

Denote by

$$
\mathcal{S}(T)=\{\lambda \in \mathbf{C}: T \text { does not have the SVEP at } \lambda\} .
$$

Corollary 3.11. Let $T \in \mathcal{B}(X)$.

- $\sigma_{g k}(T) \cup \mathcal{S}(T)=\sigma_{l g D}(T)$.

- $\sigma_{g k}(T) \cup \mathcal{S}\left(T^{*}\right)=\sigma_{r g D}(T)$.

- If $T^{*}$ has SVEP, then $\sigma_{l g D}(T)=\sigma_{g D}(T)$ and $\sigma_{g k}(T)=\sigma_{r g D}(T)$.

- If $T$ has SVEP, then $\sigma_{r g D}(T)=\sigma_{g D}(T)$ and $\sigma_{g k}(T)=\sigma_{l g D}(T)$.

- If $X$ is a Hilbert space and $T$ is a self-adjoint operator, then $\sigma_{l g D}(T)=$ $\sigma_{r g D}(T)=\sigma_{g D}(T)$.

Similarity, for operators of Kato type we have,

Corollary 3.12. Let $T \in \mathcal{B}(X)$.

- $\sigma_{k}(T) \cup \mathcal{S}(T)=\sigma_{l D}(T)$.

- $\sigma_{k}(T) \cup \mathcal{S}\left(T^{*}\right)=\sigma_{r D}(T)$.

- If $T^{*}$ has SVEP, then $\sigma_{l D}(T)=\sigma_{D}(T)$ and $\sigma_{k}(T)=\sigma_{r D}(T)$.

- If $T$ has SVEP, then $\sigma_{r D}(T)=\sigma_{D}(T)$ and $\sigma_{k}(T)=\sigma_{l D}(T)$.

In the following, we show that both $\sigma_{l g D}(T)$ and $\sigma_{r g D}(T)$ are stable under additive commuting finite rank operators.

Proposition 3.13. Let $T \in \mathcal{B}(X)$ and $F$ is a finite rank operator on $X$ such that $T F=F T$. Then $\sigma_{l g D}(T+F)=\sigma_{l g D}(T)$. 
Proof. From [18, Lemma 2.3] we know that $a c c \sigma_{a p}(T+F)=a c c \sigma_{a p}(T)$. Then $\lambda \notin a c c \sigma_{a p}(T+F)$ if and only if $\lambda \notin a c c \sigma_{a p}(T)$. Hence $\lambda I-(T+F)$ is left generalized Drazin invertible if and only if $\lambda I-T$ is left generalized Drazin invertible. So $\sigma_{l g D}(T+F)=\sigma_{l g D}(T)$.

As a consequence of Proposition 3.13 we have

Proposition 3.14. Let $T \in \mathcal{B}(X)$ and $F$ is a finite rank operator on $X$ such that $T F=F T$. Then $\sigma_{r g D}(T+F)=\sigma_{r g D}(T)$.

Proposition 3.15. Let $T \in \mathcal{B}(X)$ and $0 \in \rho(T)$. Then $\lambda \in \sigma_{l g D}(T)$ if and only if $\lambda \neq 0$ and $\lambda^{-1} \in \sigma_{l g D}\left(T^{-1}\right)$.

Proof. we have

$$
\lambda I-T=-\lambda\left(\lambda^{-1} I-T^{-1}\right) T .
$$

Since $0 \in \rho(T)$ and $T$ commute with $\left(\lambda^{-1}-T^{-1}\right)$, it follows that $\left(\lambda^{-1} I-\right.$ $\left.T^{-1}\right)_{M}$ is bounded below if and only if $(\lambda I-T)_{M}$ is bounded below and $\left(\lambda^{-1} I-T^{-1}\right)_{N}$ is quasinilpotent if and only if $(\lambda I-T)_{N}$ is quasinilpotent. This is equivalent to the statement of the Theorem.

Theorem 3.16. Let $T, S \in \mathcal{B}(X)$. If $0 \in \rho(T) \cap \rho(S)$, such that $T^{-1}-S^{-1}$ is finite rank operator commuting with $T$ or $S$, then

$$
\sigma_{l g D}(T)=\sigma_{l g D}(S)
$$

Proof. Proposition 3.13 implies that $\sigma_{l g D}\left(T^{-1}\right)=\sigma_{l g D}\left(S^{-1}\right)$, and by Proposition 3.15 we have $\sigma_{l g D}(T)=\sigma_{l g D}(S)$.

Proposition 3.17. Let $T \in \mathcal{B}(X)$ and $0 \in \rho(T)$. Then $\lambda \in \sigma_{r g D}(T)$ if and only if $\lambda \neq 0$ and $\lambda^{-1} \in \sigma_{r g D}\left(T^{-1}\right)$.

Theorem 3.18. Let $T, S \in \mathcal{B}(X)$. If $0 \in \rho(T) \cap \rho(S)$, such that $T^{-1}-S^{-1}$ is a finite rank operator commuting with $T$ or $S$, then

$$
\sigma_{r g D}(T)=\sigma_{r g D}(S)
$$

Theorem 3.19. Let $R, T, U \in \mathcal{B}(X)$ be such that $T R T=T U T$. Then $\sigma_{l g D}(T R)=\sigma_{l g D}(U T)$. 
Proof. Since $\sigma_{a p}(T R) \backslash\{0\}=\sigma_{a p}(U T) \backslash\{0\}$, from [5, Theorem 1], then it is enough to show that $T R$ is left generalized Drazin invertible if anf only if $U T$ is. Assume that $0 \notin \sigma_{l g D}(T R)$, then $0 \in i s o \sigma_{a p}(T R)$. Therefore $T R-\mu I$ is bounded below for all small $\mu \neq 0$. Hence $U T-\mu I$ is bounded below for all small $\mu \neq 0$. So $0 \in i s o \sigma_{a p}(U T)$. Hence $U T$ is left generalized Drazin invertible if and only if $T R$ is left generalized Drazin invertible.

By duality, we have:

Theorem 3.20. Let $R, T, U \in \mathcal{B}(X)$ be such that $T R T=T U T$. Then $\sigma_{r g D}(T R)=\sigma_{r g D}(U T)$.

In particular if $R=U$ we get

Corollary 3.21. Let $R, T \in \mathcal{B}(X)$ then $\sigma_{l g D}(T R)=\sigma_{l g D}(R T)$ and $\sigma_{r g D}(T R)=$ $\sigma_{r g D}(R T)$.

Example 3.22. Let $R, T \in \mathcal{B}(X)$ and $A$ be the operator defined on $X \oplus X$ by

$$
A=\left(\begin{array}{cc}
0 & T \\
R & 0
\end{array}\right),
$$

then $A^{2}=\left(\begin{array}{cc}T R & 0 \\ 0 & R T\end{array}\right)=T R \oplus R T$. Thus $\sigma_{l g D}\left(A^{2}\right)=\sigma_{l g D}(T R) \cup$ $\sigma_{l g D}(R T)$ which equals to $\sigma_{l g D}(T R)$ from Corollary 3.21. Therefore $\sigma_{l g D}(A)=$ $\left(\sigma_{l g D}(T R)\right)^{1 / 2}$. Similarly we have $\sigma_{r g D}(A)=\left(\sigma_{r g D}(T R)\right)^{1 / 2}$.

\section{Generalized Drazin inverse and local spectral theory}

We know that if $T \in \mathcal{B}(X)$ is not invertible then $T$ is generalized Drazin invertible if and only if $X=K(T) \oplus H_{0}(T)$ and, with respect tho this decomposition, $\mathrm{T}=\mathrm{T}_{1} \oplus T_{2}$, with $T_{1}=T_{K(T)}$ is invertible and $T_{2}=T_{H_{0}(T)}$ is quasinilpotent.

Note that the generalized Drazin inverse $T^{D}$ of $T$, if it exists, is uniquely determined and represented, with respect of the same decomposition, as the direct sum $\mathrm{T}^{D}=T_{1}^{-1} \oplus 0$, with $T_{1}^{-1}$ is the inverse of $T_{1}$, Furthermore, the nonzero part of the spectrum of $T^{D}$ is given by the reciprocals of the nonzero points of the spectrum of $T$, i.e.,

$$
\sigma\left(T^{D}\right) \backslash\{0\}=\left\{\frac{1}{\lambda}: \lambda \in \sigma(T) \backslash\{0\}\right\} .
$$

Since the spectral mapping theorem holds for the approximate spectrum and the surjective spectrum, we have $\sigma_{a p}\left(T^{D}\right) \backslash\{0\}=\left\{\frac{1}{\lambda}: \lambda \in \sigma_{a p}(T) \backslash\{0\}\right\}$ and $\sigma_{s u}\left(T^{D}\right) \backslash\{0\}=\left\{\frac{1}{\lambda}: \lambda \in \sigma_{s u}(T) \backslash\{0\}\right\}$. 
An interesting question given in [2] is that there is a reciprocal relationship between the nonzero part of the local spectrum of a Drazin invertible operator and the nonzero part of the local spectrum of its Drazin inverse. In the sequel we study this question in the case of the generalized Drazin invertible operators.

Before this down, we shall give the relevant definitions concerning the local spectral theory. Given a bounded linear operator $T \in \mathcal{B}(X)$, the local resolvent set $\rho_{T}(x)$ of $T$ at a point $x \in X$ is defined as the union of all open subsets $U$ of $\mathbf{C}$ such that there exists an analytic function $f: U \longrightarrow X$ satisfying

$$
(\lambda I-T) f(\lambda)=x \quad \text { for all } \lambda \in U .
$$

The local spectrum $\sigma_{T}(x)$ of $T$ at $x$ is the set defined by $\sigma_{T}(x):=$ $\mathbf{C} \backslash \rho_{T}(x)$. Obviously, $\sigma_{T}(x) \subseteq \sigma(T)$.

The SVEP for $T$ is equivalent to saying that $\sigma_{T}(x)=\emptyset$ if and only if $x=0$, see [19, Proposition 1.2.16]. Note that if $T$ has SVEP then a spectral theorem holds for the local spectrum, i.e., if $f$ is an analytic function defined on an open neighborhood $U$ of $\sigma(T)$ then

$$
f\left(\sigma_{T}(x)\right)=\sigma_{f(T)}(x) \quad \text { for all } x \in X .
$$

See also $[21]$.

An important invariant subspace in local spectral theory is given by the local spectral subspace of $T$ associated at a subset $\Omega \subseteq \mathbf{C}$, defined as

$$
X_{T}(\Omega)=\left\{x \in X: \sigma_{T}(x) \subset \Omega\right\} .
$$

Obviously, for every closed set $\Omega \subseteq \mathbf{C}$ we have

$$
X_{T}(\Omega)=X_{T}(\Omega \cap \sigma(T)) .
$$

For a closed subset $\Omega \subseteq \mathbf{C}$, the glocal subspace $\mathcal{X}_{T}(\Omega)$ is defined as the set of all $x \in X$ for which there exists an analytic function $f: \mathbf{C} \backslash \Omega \longrightarrow X$ satisfying $(\lambda I-T) f(\lambda)=x$ on $\mathbf{C} \backslash \Omega$.

Obviously, for $\Omega$ a closed set, $\mathcal{X}_{T}(\Omega) \subset X_{T}(\Omega)$, and we have equality when $T$ satisfies the SVEP.

Definition 4.1. An operator $T \in \mathcal{B}(X)$ is said to have Dunford's property $(C)$, shortly property $(C)$, if $X_{T}(\Omega)$ is closed for every closed set $\Omega \subseteq \mathbf{C}$.

Definition 4.2. An operator $T \in \mathcal{B}(X)$ is said to have property $(Q)$, if $H_{0}(\lambda I-T)$ is closed for every $\lambda \in \mathbf{C}$. 
Another important property which plays a central role in local spectral theory is the following one introduced by Bishop, see [19]:

Definition 4.3. An operator $T \in \mathcal{B}(X)$ is said to have Bishop's property $(\beta)$, shortly property $(\beta)$, if for every open set $\mathcal{U}$ of $\mathbf{C}$ and every sequence of analytic functions $f_{n}: \mathcal{U} \longrightarrow X$ for which $(\lambda I-T) f_{n}(\lambda) \rightarrow 0$ uniformly on all compact subsets of $\mathcal{U}$; then also $f_{n}(\lambda) \rightarrow 0$, again locally uniformly on $\mathcal{U}$.

We have

$$
\text { property }(\beta) \Rightarrow \operatorname{property}(C) \Rightarrow \text { property }(Q) \Rightarrow \text { the SVEP . }
$$

See the monograph [19] for a detailed study of these properties.

The next first result shows that the SVEP is transmitted from $T$ to its generalized Drazin inverse $T^{D}$,

Theorem 4.4. Let $T \in \mathcal{B}(X)$ be generalized Drazin invertible. Then $T$ has the SVEP if and only if $T^{D}$ has the SVEP.

Proof. Suppose that $T \in \mathcal{B}(X)$ is a generalized Drazin invertible. If $0 \notin \sigma(T)$. Then $f(\lambda)=\frac{1}{\lambda}$ is analytic in any open neighborhood of $\sigma(T)$ which does not contains 0 , so by [19, Theorem 3.3.6] $T^{D}=T^{-1}=f(T)$ has the property SVEP. Now if $0 \in \sigma(T)$. Then $T=T_{1} \oplus T_{2}$ with $T_{1}$ is invertible and $T_{2}$ is quasinilpotent. From the first case $T_{1}$ has the property SVEP, $T_{2}$ has also the SVEP because it is quasinilpotent. So the Drazin generalized inverse $T^{D}=T_{1}^{-1} \oplus 0$ has the SVEP, from [1, Theorem 2.9].

Conversely; if $T^{D}=T_{1}^{-1} \oplus 0$ has the SVEP then $T_{1}^{-1}$ and $T_{1}$ have the property SVEP. Consequently, again by [1, Theorem 2.9], $T=T_{1} \oplus T_{2}$ has the property SVEP.

In the following result, we show that the relation equ:opinv holds also in the local sens.

Theorem 4.5. Let $T \in \mathcal{B}(X)$ be generalized Drazin invertible with generalized Drazin inverse $T^{D}$. If $T$ has the $S V E P$, then for every $x \in X$ we have

$$
\sigma_{T^{D}}(x) \backslash\{0\}=\left\{\frac{1}{\lambda}: \lambda \in \sigma_{T}(x) \backslash\{0\}\right\}
$$


Proof. Suppose that $T$ has the SVEP. If $0 \notin \sigma(T)$ then $T^{D}=T^{-1}$ and the equality equ:locspec follows from the spectral mapping theorem $[21,1.6]$ applied to the function $f(\lambda)=\frac{1}{\lambda}$. Suppose that $0 \in \sigma(T)$. According the decomposition $X=K(T) \oplus H_{0}(T), T_{1}=T_{K(T)}$ is invertible and $T_{2}=T_{H_{0}(T)}$ is quasinilpotent, then the restrictions $T_{1}$ and $T_{2}$ have the SVEP. Now, let $x \in X$ and write $x=y+z$, with $y \in K(T)$ and $z \in H_{0}(T)$. Then by [1, Theorem 2.9] we have

$$
\sigma_{T}(x)=\sigma_{T_{1}}(y) \cup \sigma_{T_{2}}(z) .
$$

The generalized Drazin inverse $T^{D}=T_{1}^{-1} \oplus 0$ has the SVEP, so always by [1, Theorem 2.9] we have

$$
\sigma_{T^{D}}(x)=\sigma_{T_{1}^{-1}}(y) \cup \sigma_{0}(z)
$$

where

$$
\sigma_{T_{1}^{-1}}(y)=\left\{\frac{1}{\lambda}: \lambda \in \sigma_{T_{1}}(y)\right\} \quad \text { for all } y \in K(T) .
$$

In the case $z=0, \sigma_{T_{2}}(0)=\emptyset$ and hence $\sigma_{T}(x)=\sigma_{T_{1}}(y)$ and, analogously, $\sigma_{T^{D}}(x)=\sigma_{T_{1}^{-1}}(y)$. Thus equ:locspec. Now, if $z \neq 0, \sigma_{T_{2}}(z)=\{0\}=$ $\sigma_{0}(z)$, since both $T_{2}$ and the null operator are quasinilpotent operators. Furthermore, $0 \notin \sigma_{T_{1}}(y)$ and $0 \notin \sigma_{T_{1}^{-1}}(y)$, hence $\sigma_{T}(x) \backslash\{0\}=\sigma_{T_{1}}(y)$ and $\sigma_{T^{D}} \backslash\{0\}(x)=\sigma_{T_{1}^{-1}}(y)$, from which we deduce,

$$
\sigma_{T^{D}}(x) \backslash\{0\}=\sigma_{T_{1}^{-1}}(y)=\left\{\frac{1}{\lambda}: \lambda \in \sigma_{T_{1}}(y)\right\}=\left\{\frac{1}{\lambda}: \lambda \in \sigma_{T}(x) \backslash\{0\}\right\} .
$$

This complete the proof.

We establish now that also the property $(C)$ is transferred to the generalized Drazin inverse.

Theorem 4.6. Let $T \in \mathcal{B}(X)$ be a generalized Drazin invertible. Then $T$ has the property $(C)$ if and only if $T^{D}$ the property $(C)$.

Proof. Suppose that $T \in \mathcal{B}(X)$ is generalized Drazin invertible. If $0 \notin \sigma(T)$, then $f(\lambda)=\frac{1}{\lambda}$ is analytic in any open neighborhood of $\sigma(T)$ which does not contains 0 , so by $\left[19\right.$, Theorem 3.3.6] $T^{D}=T^{-1}=f(T)$ has the property $(C)$. Now if $0 \in \sigma(T)$. Then $T$ admits a $\operatorname{GKD}(M, N)$, with $M=K(T)$ and $N=H_{0}(T), T_{M}$ is invertible and $T_{N}$ is quasinilpotent. For the Drazin generalized inverse $T^{D}=T_{M}^{-1} \oplus 0$, we have

$$
X_{T^{D}}(\Omega)=M_{T_{M}^{-1}}(\Omega) \oplus N_{0}(\Omega) \text { for every closed set } \Omega \subseteq \mathbf{C} .
$$


Since $T_{M}$ is invertible, by the first case, $T_{M}$ has the property $(C)$ and so the inverse $T_{M}^{-1}$ has the property $(C)$ with $M_{T_{M}^{-1}}(\Omega)$ is closed for every closed set $\Omega \subseteq$ C. We know that $N_{0}(\Omega)=\{0\}$ if $0 \notin \Omega$ and $N_{0}(\Omega)=N$ if $0 \in \Omega$. Then, $X_{T^{D}}(\Omega)=M_{T_{M}^{-1}}(\Omega) \oplus\{0\}$ if $0 \notin \Omega$ and $X_{T^{D}}(\Omega)=$ $M_{T_{M}^{-1}}(\Omega) \oplus N$ if $0 \in \Omega$. In both cases $X_{T^{D}}(\Omega)$ is closed, and consequently $T^{D}$ has the property $(C)$.

Conversely; if $T^{D}=T_{M}^{-1} \oplus 0$ has the property $(C)$ and as above $X_{T^{D}}(\Omega)=M_{T_{M}^{-1}}(\Omega) \oplus\{0\}$ if $0 \notin \Omega$ and $X_{T^{D}}(\Omega)=M_{T_{M}^{-1}}(\Omega) \oplus N$ if $0 \in \Omega$. This implies that $M_{T_{M}^{-1}}(\Omega)$ is closed and hence $T_{M}^{-1}$ has the property $(C)$. Thus $T_{M}$ has the property $(C)$. Since $X_{T}(\Omega)=M_{T_{M}}(\Omega) \oplus N_{T_{N}}(\Omega)$, and $T_{N}$ is quasinilpotent it then follows that $X_{T}(\Omega)=M_{T_{M}}(\Omega) \oplus\{0\}$ if $0 \notin \Omega$, or $X_{T}(\Omega)=M_{T_{M}}(\Omega) \oplus N$ if $0 \in \Omega$. Therefore $X_{T}(\Omega)$ is closed for every closed set $\Omega \subseteq \mathbf{C}$. Thus $T$ has the property $(C)$.

Since for operator having property $(Q)$ we have

$$
H_{0}(\lambda I-T)=X_{T}(\{\lambda\})=\mathcal{X}_{T}(\{\lambda\}) \text { for all } \lambda \in \mathbf{C},
$$

we can deduce from Theorem 4.6 that

Corollary 4.7. Let $T \in \mathcal{B}(X)$ be generalized Drazin invertible. Then $T$ has the property $(Q)$ if and only if $T^{D}$ has the property $(Q)$.

Now before to study the property $(\beta)$, we need some preliminary results. Let $H(U, X)$ denote the space of all analytic functions from $U$ into $X$. With respect to pointwise vector space operations and the topology of locally uniform convergence, $H(U, X)$ is a Fréchet space. For every $T \in \mathcal{B}(X)$ and every open set $U \subseteq \mathbf{C}$, define $T_{U}: H(U, X) \longrightarrow H(U, X)$ by

$$
\left(T_{U} f\right)(\lambda):=(\lambda I-T) f(\lambda) \quad \text { for all } f \in H(U, X) \text { and } \lambda \in U \text {. }
$$

From [19, Proposition 3.3.5], $T$ has the property $(\beta)$ if and only if for every open set $U \subseteq \mathbf{C}$, the operator $T_{U}$ has closed range in $H(U, X)$. Evidently, the property $(\beta)$ is inherited by the restrictions on invariant closed subspaces. Furthermore, the following theorem shows that the property $(\beta)$ is transmitted reciprocally form a generalized Drazin invertible operator to its generalized Drazin inverse.

Theorem 4.8. Let $T \in \mathcal{B}(X)$ a generalized Drazin invertible. Then $T$ has the property $(\beta)$ if and only if $T^{D}$ has the property $(\beta)$. 
Proof. Suppose that $T \in \mathcal{B}(X)$ is generalized Drazin invertible and $0 \in \sigma(T)$. Then $T=T_{1} \oplus T_{2}$ with $T_{1}$ is invertible and $T_{2}$ is quasinilpotent. From [19, Proposition 2.1.6], we can identify $H(U, X)$ with the direct sum $H(U, K(T)) \oplus H\left(U, H_{0}(T)\right) . T_{1}$ has the property $(\beta)$ and hence its inverse $T_{1}^{-1}$ has the property $(\beta)$. Now $\mathrm{T}_{U}^{D}[H(U, X)]=\left(T_{1}^{-1} \oplus 0_{U}\right)[H(U, K(T)) \oplus$ $\left.H\left(U, H_{0}(T)\right)\right]$ $=\left(T_{1}^{-1}\right)_{U}[H(U, K(T))] \oplus 0_{U}\left[H\left(U, H_{0}(T)\right)\right]$.

Clearly $T_{U}^{D}$ has closed range in $H(U, X)$, so $T^{D}$ has the property $(\beta)$.

Conversely; if $T^{D}=T_{1}^{-1} \oplus 0$ has the property $(\beta)$. Then as above $T_{1}$ has the property $(C)$. Since the quasinilpotent operator $T_{2}$ has the property $(\beta)$ and the fact that

$$
\mathrm{T}_{U}[H(U, X)]=\left(T_{1} \oplus T_{2}\right)_{U}\left[H(U, K(T)) \oplus H\left(U, H_{0}(T)\right)\right]
$$

$=T_{1 U}[H(U, K(T))] \oplus T_{2 U}\left[H\left(U, H_{0}(T)\right)\right]$,

we conclude that $T_{U}^{D}$ has closed range in $H(U, X)$, so $T^{D}$ has the property $(\beta)$.

An operator $T \in \mathcal{B}(X)$ is said to have the decomposition property $(\delta)$ if the decomposition

$$
X=X_{T}(\bar{U})+X_{T}(\bar{V})
$$

holds for every open cover $\{U, V\}$ of $\mathbf{C}$. Note that $T \in \mathcal{B}(X)$ has property $(\delta)$ (respectively, property $(\beta))$ if and only if $T^{*}$ has property $(\beta)$ (respectively, property $(\delta)$ ), see [19, Theorem 2.5.5]. If $T \in \mathcal{B}(X)$ has both property $(\beta)$ and property $(\delta)$ then $T$ is said to be decomposable.

Corollary 4.9. Suppose that $T$ is generalized Drazin invertible. If $T$ has property $(\delta)$ then $T^{D}$ has property $(\delta)$, and analogously, if $T$ is decomposable then $T^{D}$ is decomposable.

Proof. Clearly, from the definition of the generalized Drazin invertibility it follows that if $T$ is generalized Drazin invertible then its adjoint $T^{*}$ is also generalized Drazin invertible, with Drazin inverse $T^{D *}$. If $T$ has property $(\delta)$ then $T^{*}$ has property $(\beta)$ and hence, by Theorem 4.8 , also $T^{D *}$ has property $(\beta)$. By duality this implies that $T^{D}$ has property $(\delta)$. The second assertion is clear: if $T$ is decomposable then $T^{D}$ has both properties $(\delta)$ and $(\beta)$ and the same holds for $T^{D}$, again by Theorem 4.8 and the first part of the proof. Hence $T^{D}$ is decomposable. 
A natural question suggested by all the results of this section is whether the local spectral properties are transmitted from a left (resp. right) generalized Drazin invertible operator to its left (resp. right) generalized Drazin inverse. The next example shows that the answer to this question is negative.

Example 4.10. Let $X=\ell^{2}$ be the Hilbert space of all square summable complex sequences

$$
x=\left(x_{n}\right)_{n}=\left(x_{1}, x_{2}, \ldots\right),
$$

indexed by the a nonnegative integers. We define the right shift operator $R$ and the left shift operator $L$ in $\ell^{2}$ by

$$
R\left(x_{1}, x_{2}, \ldots\right)=\left(0, x_{1}, x_{2}, \ldots\right)
$$

and

$$
L\left(x_{1}, x_{2}, \ldots\right)=\left(x_{2}, x_{3}, \ldots\right) .
$$

We know that $\sigma(R)=\sigma(L)=\mathbf{D}=\{\lambda \in \mathbf{C} ;|\lambda| \leq 1\}$ and $L=R^{*}$. Furthermore, $R$ is injective with colsed range and $L$ is surjective. So $R$ is left invertible with $L$ its left inverse. Similarity, $L$ is right invertible with $R$ its right inverse.

Now, from [19, Example 1.2.8], it follows that the unilateral right shift $R$ has the property $(\beta)$ (hence has the property $(C)$, the property $(Q)$ and has the SVEP), while $L$ fails to have the SVEP, see [19, Proposition 1.2.10].

We also have

$$
\sigma_{R}(x)=\sigma(R)
$$

for every $x \in X$, so $\sigma_{R}(x) \backslash\{0\}$ is the punctured disc $\mathbf{D} \backslash\{0\}$. Consequently, the points of $\sigma_{L}(x) \backslash\{0\}$, for any left inverse $L$, cannot be the reciprocals of $\sigma_{R}(x) \backslash\{0\}$, otherwise $\sigma_{L}(x)$, and hence $\sigma(L)$, would be unbounded.

By the same notations of the definitions 2.1 and 2.2 and from [19, Proposition 1.2.10] we deduce that:

Proposition 4.11. Let $T \in \mathcal{B}(X)$. We have

- If $T$ is right generalized Drazin invertible and $T_{K(T)}$ has the SVEP (respectively, property $(C)$, property $(Q)$, property $(\beta)$ ), then $T$ is generalized Drazin invertible.

- If $T$ is left generalized Drazin invertible and $T_{K\left(T^{*}\right)}^{*}$ has the SVEP (respectively, property $(C)$, property $(Q)$, property $(\beta)$ ), then $T$ is generalized Drazin invertible. 


\section{References}

[1] P. Aiena, Fredholm and local spectral theory, with applications to multipliers. Dordrecht: Springer, 2004, doi: 10.1007/1-4020-2525-4.

[2] P. Aiena and S. Triolo, "Local spectral theory for Drazin invertible operators", Journal of mathematical analysis and applications, vol. 435, no. 1, pp. 414-424, Mar. 2016, doi: 10.1016/j.jmaa.2015.10.042.

[3] S. Campbell, Singular systems of differential equations, vol. 1. London: Pitman, 1980.

[4] S. Campbell, Singular systems of differential equations, vol. 2. London: Pitman, 1982.

[5] G. Corach, B. Duggal, and R. Harte, "Extensions of Jacobsons Lemma", Communications in algebra, vol. 41, no. 2, pp. 520-531, Feb. 2013, doi: 10.1080/00927872.2011.602274.

[6] M. Drazin, "Pseudo-inverses in associative rings and semigroups", The American mathematical monthly, vol. 65, no. 7, pp. 506-514, Sep. 1958, doi: 10.1080/00029890.1958.11991949.

[7] Q. Jiang , H. Zhong, "Generalized Kato decomposition, single-valued extension property and approximate point spectrum", Journal of mathematical analysis and applications, vol. 356, no. 1, pp. 322-327, Aug. 2009, doi: 10.1016/j.jmaa.2009.03.017.

[8] M. Cvetković, "On upper and lower generalized Drazin invertible operators", Functional analysis, approximation and computation, vol. 7, no. 3, pp. 67-74, 2015. [On line]. Available: https://bit.ly/2PoFRoe

[9] M. González, M. Mbekhta and M. Oudghiri, "On the isolated points of the surjective spectrum of bounded operator", Proceedings of the American mathematical society, vol. 136, no. 10, pp. 3521-3528, May 2008, doi: 10.1090/S0002-9939-08-09549-X.

[10] R. Harte, "Spectral projections", Irish mathematical society newsletter, vol. 11, pp. 10-15, Sep. 1984. [On line]. Available: https://bit.ly/36z8ll1

[11] M. Kaashoek and D. Lay, "Ascent, descent, and commuting perturbations", Transactions of the American mathematical society, vol. 169, pp. 35-47, 1972, doi: 10.1090/S0002-9947-1972-0312299-8.

[12] T. Kato, Perturbation theory for linear operators, New York: Springer, 1966.

[13] N. Khaldi, M. Benharrat, B. Messirdi, "Linear boundary value problems described by Drazin invertible operators", Mathematical notes, vol. 101, no. 5-6, pp. 994-999, Jun. 2017, doi: $10.1134 /$ S0001434617050261. 
[14] J. Koliha, "A generalized Drazin inverse", Glasgow mathematical journal, vol. 38, no. 3, pp. 367-381, Sep. 1996, doi: 10.1017/S0017089500031803.

[15] J. Koliha and T. Tran, "Semistable operators and singularly perturbed differential equations", Journal of mathematical analysis and applications, vol. 231, no. 2, pp. 446-458, Mar. 1999, doi: 10.1006/jmaa.1998.6235.

[16] J. Koliha and T. Tran, "The Drazin inverse for closed linear operators and the asymptotic convergence of C0-semigroups", Journal of operator theory, vol. 46, no. 2, pp. 323-336, 2001. [On line]. Available: https://bit.ly/2RVZx4u

[17] J. Labrousse, "Les opérateurs quasi-Fredholm une généralisation des opérateurs semi-Fredholm", Rendiconti del circolo matematico di Palermo, vol. 2, no. 29, pp. 161-258, May 1980, doi: 10.1007/BF02849344

[18] M. Lahrouz, M. Zohry "Weyl type theorems and the approximate point spectrum", Bulletin - Irish mathematical society, no, 55, pp. 41-51, (2005). [On line]. Available: https://bit.ly/2LYJqzg

[19] K. Laursen and M. Neumann, An introduction to local spectral theory. Oxford: Clarendon Press, 2000.

[20] K. Hocine, M. Benharrat and B. Messirdi, "Left and right generalized Drazin invertible operators", Linear and multilinear algebra, vol. 63, no. 8, pp. 1635-1648, Sep. 2015, doi: 10.1080/03081087.2014.962534.

[21] P. Vrbová, "On local spectral properties of operators in Banach spaces", Czechoslovak mathematical journal, vol. 23, no. 3, pp. 483-492, 1973. [On line]. Available: https://bit.ly/2RXwVI7 